Ann. Abeille, I963, 6 (3), $20 \mathrm{I}-227$.

\title{
ÉTUDE, AU MOYEN DE RADIO-ISOTOPES, DES ÉCHANGES DE NOURRITURE ENTRE REINES, MÂLES ET OUVRIÈRES D'APIS MELLIFICA L.
}

\author{
Francine DELVERT-SALLERON
}

Station de Recherches sur l'Abeille et les Insectes sociaux, Bures-sur-Yvetle (Seine-et-Oise)

\section{SOMMAIRE}

Le présent travail vise à introduire la méthode des traceurs radioactifs dans l'étude des échanges de nourriture entre les différentes catégories d'habitants de la ruche.

Nous déterminons tout d'abord les conditions d'expérimentation optimum. Le radio-isotope utilisé est le phosphore 32 ; il est incorporé à la nourriture.

Dans les premières quarante-huit heures, il est possible de suspecter une préférence des abeilles nourrices pour leurs sœurs. Par la suite, des résultats contradictoires ont été obtenus.

Les mâles semblent, dans certaines conditions, susceptibles de se nourrir seuls. Ils préfèrent cependant être nourris par des ouvrières.

Les ouvrières reçoivent la nourriture des mâles, soit par ingestion des régurgitations, soit par nourrissement direct.

Les reines transmettent la nourriture ou les substances contenant les traceurs radioactifs aux ouvrières; dans un groupe restreint certaines ouvrières sont privilégiées et participent plus que les autres à ces échanges.

\section{INTRODUC'TION}

Les échanges de nourriture entre les individus composant une ruche sont particulièrement intenses. Ils jouent un rôle très important dans la vie de la ruche et surtout dans la diffusion de substances comparables aux hormones et généralement désignées sous le terme de phérormone (PAIN, I96I).

Nixon et RibBands (1952) ayant fait ingérer à six ouvrières du sirop de sucre marqué au moyen de phosphore 32 , ont constaté que, sur les 24500 individus présents dans la ruche, 60 p. Ioo présentaient des traces très nettes de radioactivité au bout de vingt-quatre heures.

OERTEL et al. (I953) ont, de leur côté, étudié les échanges de nourriture entre les mâles et les ouvrières au moyen de sirop de sucre marqué au carbone $\mathrm{r}_{4}$. 
Au cours de leurs études du rayon d'action des butineuses, COURTOIS et LECOMTE (I958) en marquant les abeilles avec de l'or I98, ont constaté que Ioo p. Ioo des ouvrières devenaient radioactives au bout de trente-six heures.

Poursuivant des recherches dans la même direction que ces différents auteurs, nous nous sommes attachés plus spécialement à l'étude des échanges de nourriture entre les différents individus de la ruche, soit entre des individus appartenant à une même caste, soit entre des individus de caste ou de sexe différent.

Il est particulièrement indiqué d'utiliser la méthode des radio-isotopes pour l'étude des échanges de nourriture. Les transferts les plus infimes sont décelables sans exiger une observation continuelle et lirecte.

Après avoir discuté cles méthodes employées, nous exposerons successivement les travaux entrepris sur les ourrières, sur les mâles, puis sur les reines.

\section{MÉTHODES FXPÉRIMEXTAI,ES ET MA'TÉRIEL UTILISÉ}

T)ans la mesure du possible, les abejlles mises en expérience étaient d’age connu : nées en étuve. Dans d'autre cas, cependant, il s'agissait d'abeilles prélevées sur des cadres et constituant un échantillon représentatif de la pojulation diune ruche déteminée.

\section{0) Cagettrs}

Les abeilles ont été placées dans des cagettes du type Liebefeld formées d'un cadre de bois, fermé par deux vit res coulissantes. Pour les expériences port ant sur les échanges de nourriture entre ouvrières, ces cugettes ont été transformées par accolement en cagettes doubles ou triples, afin de séparer les abeilles en deux ou trois groupes; les compartinents communiquaient entre eux par des plaques de matiere plastique percées de trous de $3.75 \mathrm{~mm}$ de diamètre, de telle sorte que les abeilles puissent passer leur téte sans tultefois franchir la séparation.

Les cagettes étaient éfuipées d'un abreuvoir contenant de l'eau et d'une mangeoire garnie de candi it reines.

\section{2") Tempirature}

Les ragettes ont été placées dans une étuve à .30"C.

Pour les expériences sur les mâles, d’importantes mortalités se sont produites à cette température. Nous avons alors suivj les indications données par Matmis (1961) en les conservant à la température de $\left.2.3^{\circ}\right)^{\circ}$, c'est-í-dire à la température du laboratoire en été. I)ans ces conditions, la longévité des mâles a été nettement supérieure et le comportement des ouvrières n'a pas paru notablement perturbé.

\section{3") Nourrisseur}

Ia mourriture solide était présentée dans une petite boite de matière plastique de $4 \mathrm{~cm}$ sur 2,7 et de $7 \mathrm{~mm}$ de haut. Tans cet te boîte, la quant ité de nourriture était toujours supérieure à celle nécessairt aux abeilles.

Nous avons employé the nourriture liquide lorsque nous désirions rendre radioactif un seul insecte sams risque de contamination extérieure. Nous nous servions alors d'un nourrisseur constitué d'un pet it tube de verre placé verticalement sur un socle. Le niveau de miel ou de la solution sucrée contenant le radio-isotope est toujours suffisamment bas pour que l'abeille ne puisse polluer ses pattes, mais suffisamment haut pour qu'elle y ait accès avec le bout de sa langue (fig. $\mathbf{1}, \mathbf{2}, 3$ ).

\section{4") Choix de lisotope}

A la suite d'un certain nombre d'auteurs, nous avons choisi le phosphore 32 tel qu'il est fournipar le C. E. A. sous forme de phosphate monosodique ; il est ainsi facilement assimilable par l'organisme et aisément incorporable à la nourriture. Le phosphore 32 a une période de désintégration 


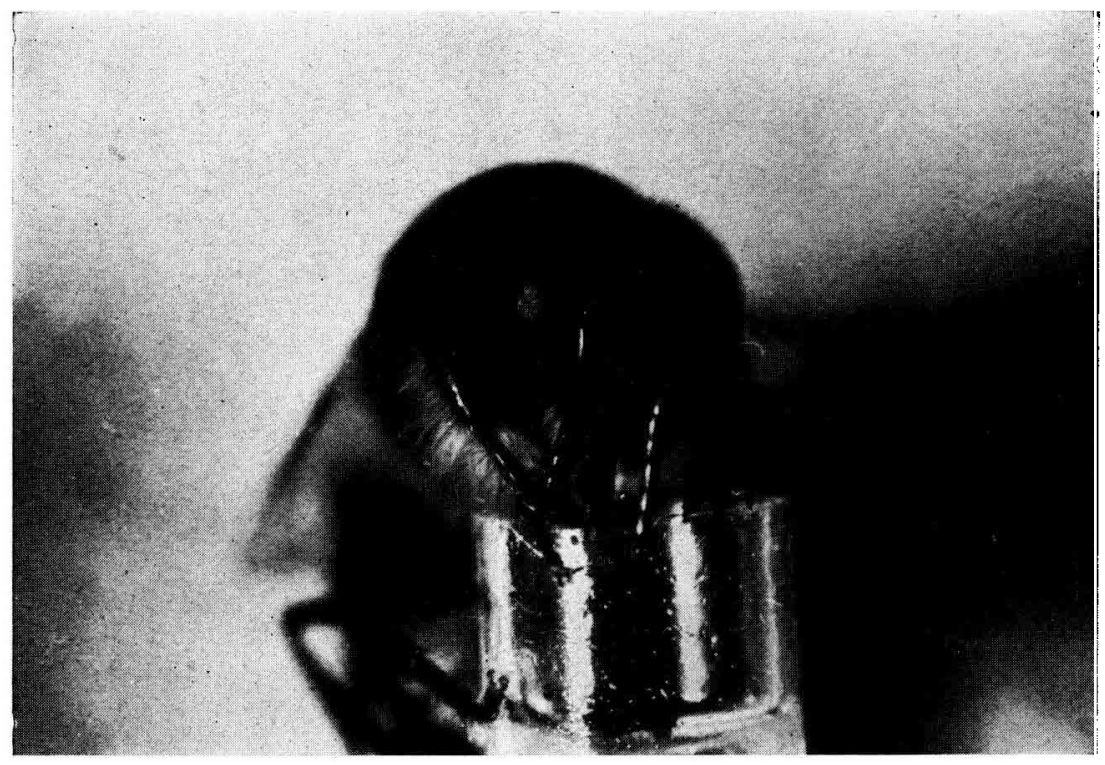

For. r. Prise to monniture par an male isolé

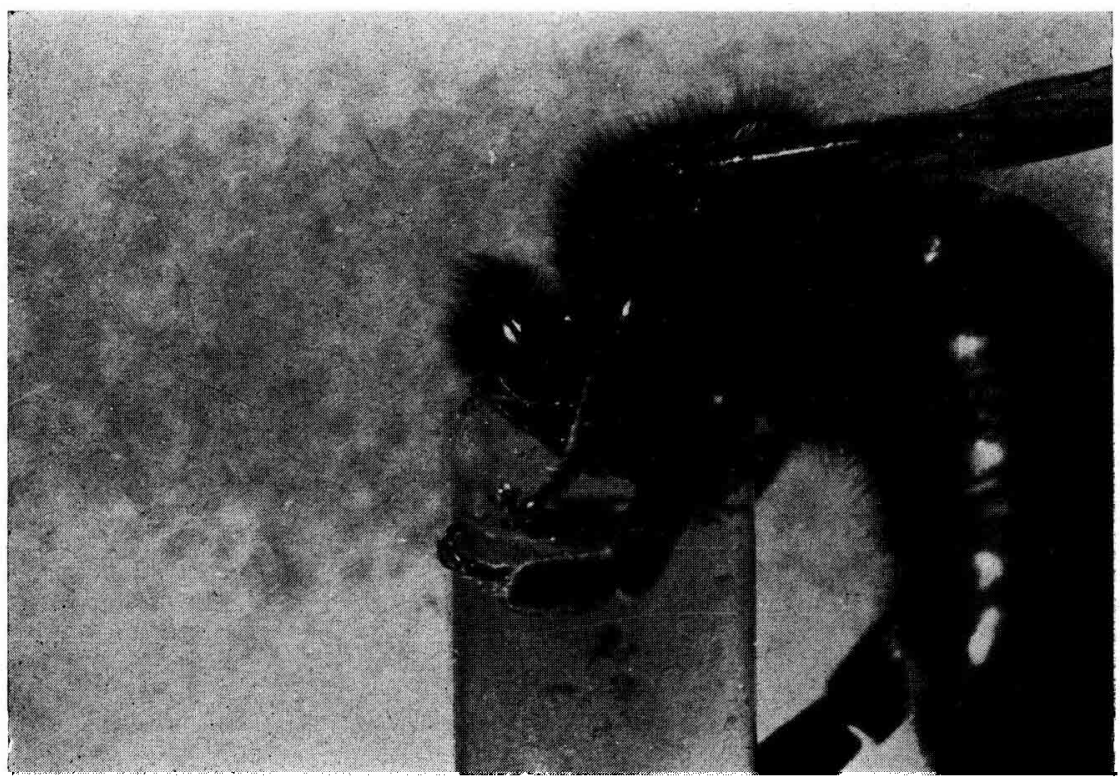

Fis. 2. La reine 
de I4,3 jours. Nous devons toutefois tenir compte de sa période biologique, c'est-à-dire de son temps moyen de séjour dans l'organisme avant élimination. On calcule la période apparente selon la formule :

$$
\frac{\mathrm{I}}{\mathrm{T}}=\frac{\mathrm{I}}{\mathrm{Tr}}+\frac{\mathrm{I}}{\mathrm{Tb}}
$$$$
\begin{aligned}
& \mathrm{T} \text { : période apparente } \\
& \mathrm{Tr} \text { : période radioactive } \\
& \mathrm{Tb} \text { : période biologique }
\end{aligned}
$$

Les auteurs considèrent généralement que la période apparente permet de travailler dans des conditions satisfaisantes.

Le phosphore $3^{2}$ se dissout facilement dans l'eau sucrée; on obtient alors une solution à taux de radioactivité homogène. Son émission de radiation $\beta$ d'énergie minimum de $\mathrm{I}, 7 \circ 7 \mathrm{MeV}$, permet une détection commode par un compteur Geiger-Müller de type cloche.

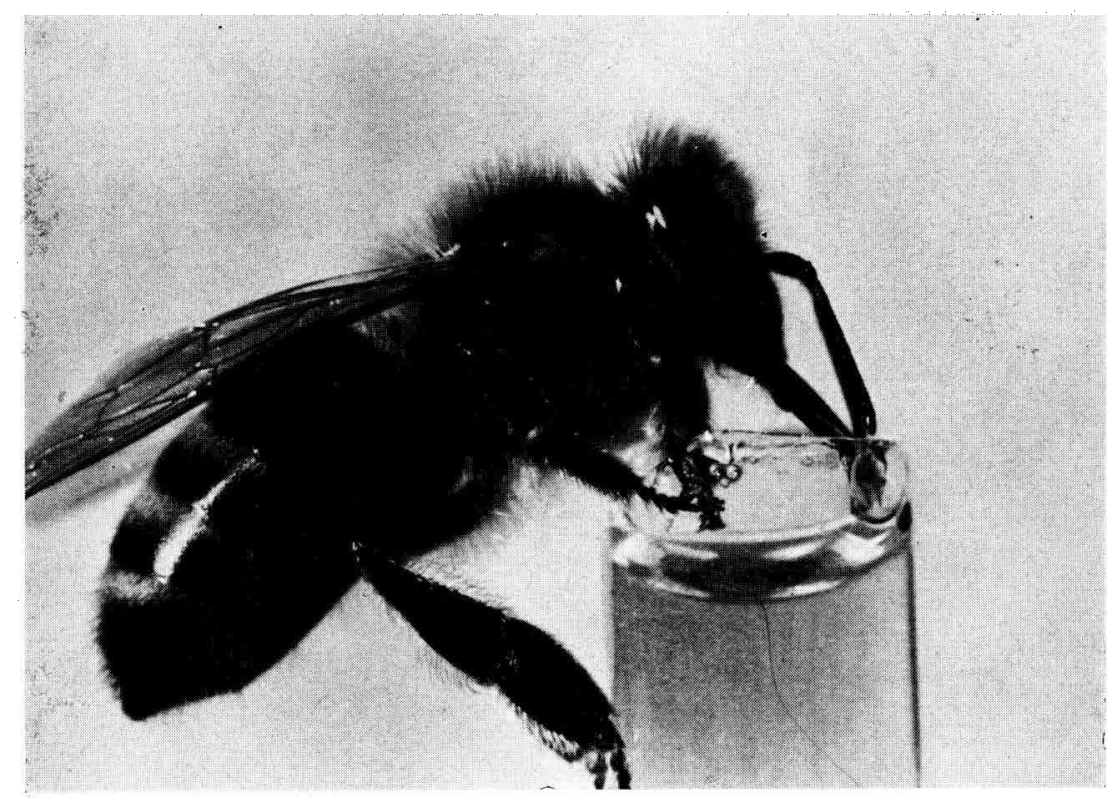

FIG. 3. - Une ouvrière

\section{5) Introduction de l'isotope}

Il y a un grave danger de manipulation à incorporer l'isotope radioactif dans le miel en train de chauffer. Pour éviter les risques de contamination au cours de la fabrication du candi, nous ne pouvons l'introduire qu'une fois le candi fait, avant qu'il ne refroidisse. Cela n'a été réalisable qu'à l'intérieur d'une boîte à gants.

Il fallait aussi s'assurer de l'homogénéité de la radioactivité de la pâte. Nous avons préparé différentes sortes de candi, en incorporant au moment du refroidissement différentes concentrations de colorants ; par exemple, un ou deux $\mathrm{cm}^{3}$ de bleu de méthylène. On obtient une pâte uniformément colorée, d'où l'on peut conclure que les substances radioactives se mélangeaient au candi avec la même homogénéité.

Cependant, pour contrôler cette homogénéité, nous avons mesuré la radioactivité de boulettes de candi de poids croissants. Le phénomène d'autoabsorption entraîne évidemment des erreurs ; par exemple, pour une boulette de $262 \mathrm{mg}$, le taux de comptage varie entre 4276 et 8 o2o coups par minute, selon que le candi est amassé ou étalé en plaque. Pour pallier cet inconvénient, il suffit de dissoudre les boulettes de poids différents dans un même volume d'eau, un $\mathrm{cm}^{3}$, contenu dans des cupules identiques. Nous obtenons alors une courbe qui confirme l'homogénéité de la préparation de candi. 


\section{$\left.6^{\circ}\right)$ Nocivité du rayonnement}

Courtors et LECOMTE (I959) ont montré qu'un rayonnement de 18 ooo Rœntgens est admissible pour l'abeille, alors que la dose admise pour l'homme est de 600 Rœntgens. Cette résistance permet d'opérer des marquages à l'aide de radioéléments artificiels d'une assez forte activité, sans risque de tuer prématurément les insectes ou de modifier leur comportement de façon appréciable.

\section{$\left.7^{\circ}\right)$ Méthode du comptage}

Nous avons employé un compteur Geiger-Müller du type cloche, fabriqué par le Laboratoire des Télécommunications : I $3 \mathrm{AP} 7$.

Son seuil de tension est de I $35^{\circ}$ volts, le palier est de $30^{\circ 0}$ volts ; nous avons travaillé avec une tension constante de I 475 volts. Le mouvement propre de l'appareil, situé dans une cave et protégé par $5 \mathrm{~cm}$ de plomb, est compris entre ro et 14 coups par minute ; ce mouvement propre (dit aussi bruit de fond) est déterminé avant chaque expérience par un comptage d'au moins to minutes.

Le tube G.-M. est placé dans un château de plomb, dans lequel on introduit les objets ou les animaux faisant l'objet de la mesure.

Les animaux sont placés près du tube dans les meilleures "conditions de géométrie ». On désigne sous le nom de " géométrie " la position de l'échantillon par rapport au tube. Il importe d'effectuer la mesure à peu de distance de la fenêtre du compteur, et de telle manière que la position de l'objet soit constante et reproductible dans les différentes expériences. L'excentrage de la source est une cause d'erreur, ainsi que la variation de la distance à la fenêtre.

Il faut bien préciser que les chiffres donnés par le compteur ne sont pas les valeurs absolues mais représentent des chiffres proportionnels à la radioactivité réelle.

C'est pourquoi dans nos résultats nous ne nous attachons pas à leur valeur absolue, mais à leur signification par rapport aux autres chiffres.

Le comptage s'opère de façons différentes selon que l'on a affaire à une abeille morte ou vivante.

L'insecte mort est introduit sous le compteur au moyen d'un porte-objet dont il est isolé par un papier-filtre, ceci pour éviter toute contamination. Une mesure du bruit de fond tous les dix comptages permet de s'assurer qu'il n'y a pas eu contamination.

L'insecte vivant, préalablement endormi au $\mathrm{CO}_{2}$, est introduit dans une petite boîte cubique en carton dont la face tournée vers le compteur est en cellophane. Cette boîte de $2 \times 2 \mathrm{~cm}$ est placée de la même façon qu'un animal mort sous le compteur. L'absorption due au couvercle de cellophane est négligeable.

Les chiffres des résultats donnés dans les différents tableaux sont exprimés en " coups nets ", c'est-à-dire après avoir déduit le bruit de fond ; nous indiquons cependant toujours ce dernier.

\section{$\left.8^{\circ}\right)$ Dissection des reines radioactives}

Pour étudier la localisation du phosphore 32 , nous avons entrepris de disséquer les cadavres de reines.

Nous avons tout d'abord opéré à sec, craignant que le phosphore ne se dissolve pas dans l'eau de la cuve. Mais les difficultés rencontrées nous ont fait abandonner cette technique, les organes adhéraient les uns aux autres et une dissection fine était à peu près impossible. Il nous a paru préférable, par la suite, de disséquer dans une solution concentrée de phosphate de sodium. Une goutte de cette solution, après emploi, passée dans le château de plomb n'était pas radioactive. Dans ces conditions, nous avons pensé que la dissolution du phosphate radioactif était insignifiante.

\section{$\left.9^{\circ}\right)$ Marquage des abeilles à la peinture}

Dans certaines expériences, il a fallu marquer les abeilles pour les reconnaître d'un jour sur l'autre.

Selon la méthode de VoN Frisch, nous les avons numérotées à l'aide de tâches colorées. La peinture utilisée sèche rapidement. Elle présente de plus l'avantage d'être fournie par petites quantités dans de multiples coloris (marque Humbrol-Plastic Enamel). En variant la couleur des taches, leur nombre et leur position, tantôt sur le thorax, tantôt sur l'abdomen, ou sur les deux, c'est avec la plus grande facilité que nous pouvons distinguer un assez grand nombre d'abeilles.

\section{Iо $\left.{ }^{\circ}\right)$ Anesthésie}

Au cours des expériences, nous avons été obligés d'endormir plusieurs fois les abeilles bien que cette anesthésie par le gaz carbonique présente des inconvénients, comme l'ont signalé de nombreux auteurs. Les abeilles sont endormies au moment de leur mise en cagette, au moment de leur marquage, et à chaque passage sous le compteur. Ceci permet d'expliquer la très forte mortalité qui nous a gêné dans un certain nombre d'expériences. 


\section{RÉSULTATS EXPÉRIMENTAUX}

\section{I. - ÉCHANGES DE NOURRITURE ENTRE OUVRIÈRES}

Comme nous l'avons déjà signalé plus haut, le mode de répartition de la nourriture à l'intérieur de la ruche a été mis en évidence par Nixon et RibBands (I952). Six abeilles sont introduites dans la ruche après avoir ingéré de la nourriture rendue radioactive par le phosphore 32 . Au bout de quatre heures, I5 p. Ioo des abeilles sont devenues radioactives, ce qui représente 62 p. Ioo des butineuses. Vingt-sept heures après le début de l'expérience, 43 p. Ioo à $60 \mathrm{p}$. Ioo des ouvrières sont radioactives.

Courtors, Lecomte et SALI,ERon (1961), lors d'une expérience analogue, ont constaté que la répartition de la nourriture se faisait à partir du "centre thermique " de la ruche en hiver.

Bien d'autres points de la biologie de l'abeille ouvrière peuvent cependant être éclaircis en utilisant la méthode des radio-isotopes; en particulier nous avons essayé de répondre à la question suivante :

\section{Les abeilles préfèrent-elles nourrir leurs sceurs plutôt que des étrangères?}

Les abeilles peuvent distinguer leurs sœurs, d'abeilles provenant d'une autre ruche. Une expérience consistant à mettre dans une cagette triple dont les compartiments sont séparés par une grille trois lots d'abeilles : un dans le compartiment central avec nourriture à sa disposition, les deux autres constitués, l'un par des abeilles venant de la même ruche, l'autre venant d'une autre ruche dans les compartiments latéraux dépourvus de nourriture, montre qu'en vingt-quatre heures la mortalité est trois fois moins importante chez les sœurs que chez les étrangères; cette différence s'annulant au bout de trois jours (KALIFMAN, I955).

Le marquage de la nourriture des abeilles du compartiment central semblait une excellente méthode pour étudier ce phénomène. Flle devrait permettre de constater si les abeilles mortes n'avaient pas été nourries et d'évaluer l'importance des échanges entre abeilles.

Pour ce gente d'expérience, nous avons employé les cagettes précédemment décrites. Seules les abeilles du compartiment central reçoivent du candi radioactif, les autres n'ont que de l'eau. Nous pouvons schématiser de la façon suivante le plan de l'expérience :

\begin{tabular}{l|c|c}
\hline \hline Sceurs & Nourrices \\
\hline & $\mathrm{I}_{\mathrm{I}}^{\mathrm{x}}$ & Étrangères \\
\hline $\mathrm{I} \longrightarrow \mathrm{I}^{\mathrm{x}}$ nourriture radioactive \\
\hline
\end{tabular}




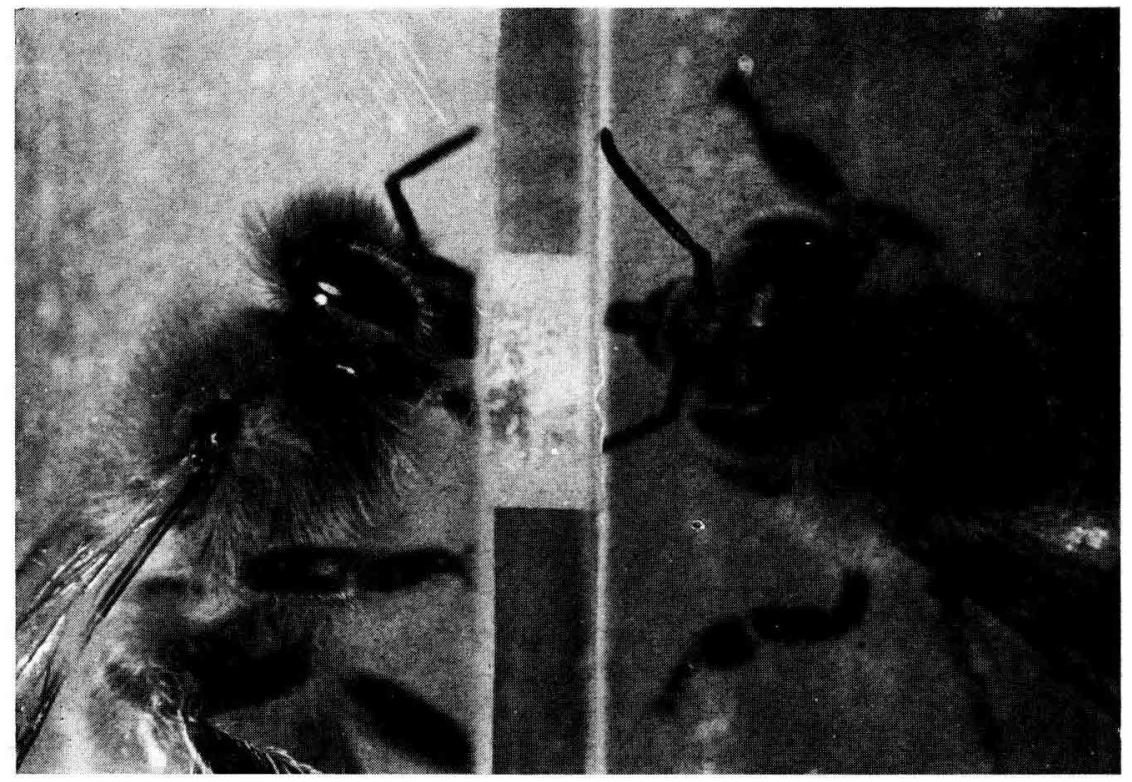

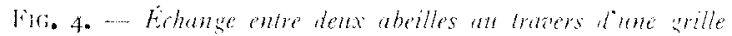

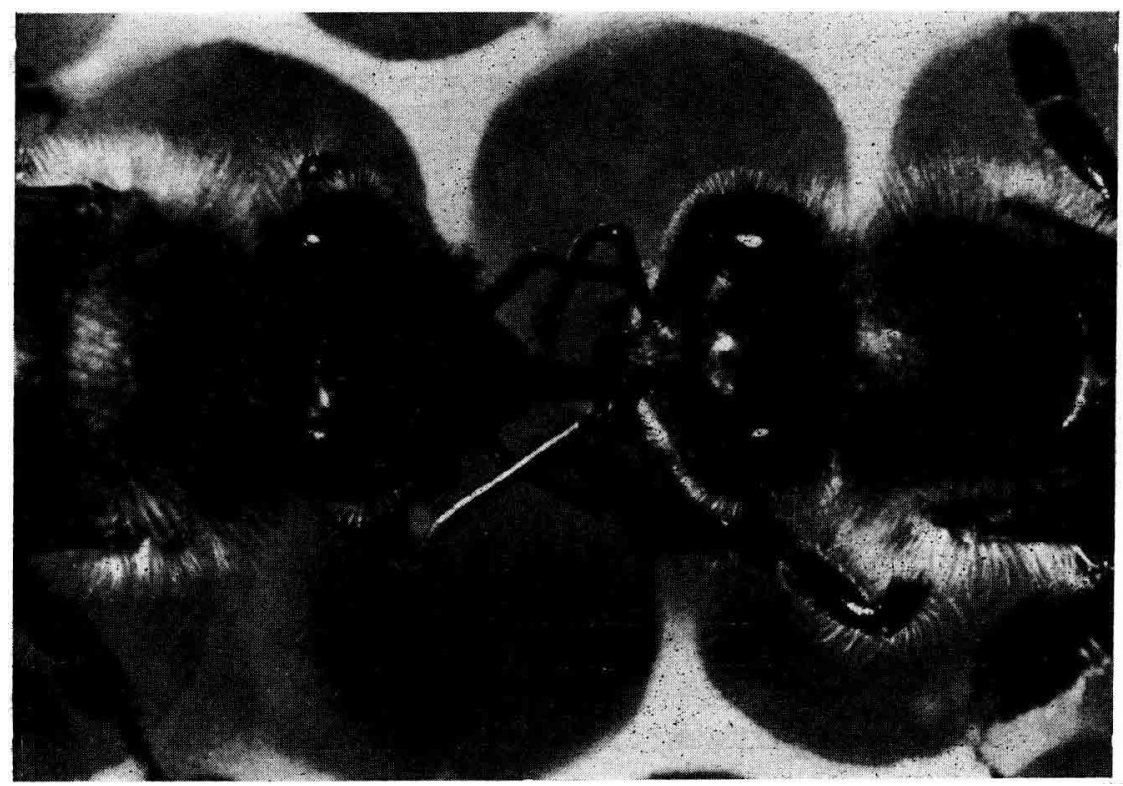

FIC. 5. - Echange entre teux absilles 
Pour la facilité de l'exposé, nous conviendrons d'appeler : nourrices, les abeilles qui reçoivent de la nourriture, sœurs, celles qui proviennent de la même ruche et, étrangères, celles qui proviennent d'une ruche différente. Les nourrices peuvent alimenter les sœurs et les étrangères. Lors de la mise en cagette, le nombre d'abeilles sœurs et le nombre d'abeilles étrangères est toujours le même. A la fin de l'expérience, après avoir compté la radioactivité des cadavres, on tue toutes les abeilles de la cagette avec de l'acétate d'éthyle, avant de mesurer la radioactivité de chaque insecte. Les résultats obtenus, très différents selon les cagettes, ne sont utilisables que pour comparer la radioactivité des lots d'une même cagette. Pour pouvoir comparer les résultats de deux essais successifs, nous avons évalué la radioactivité moyenne d'une abeille pour chaque lot, et nous l'avons convertie en pourcentage par rapport à la radioactivité moyenne d'une nourrice du même essai. Ces pourcentages nous ont donné des grandeurs comparables d'un essai à l'autre.

Un numéro a été attribué à chaque essai ; comme les lots d'abeilles présentaient des mortalités différentes, nouts avons évalué la radioactivité moyenne par lots, en ne tenant compte que des chiffres obtenus par le comptage des abeilles vivantes.

Les résultats ont été reportés sur un graphique dont le mode de représentation est le suivant :

- en abscisse est porté le nombre d'abeilles vivantes en fin d'expériences,

- en ordonnée, le pourcentage de la moyenne de la teneur en radioisotope des abeilles soeurs (S), ou étrangères (E), établi par rapport à celle des nourrices.

Par exemple, dans l'essai no 3 , à la fin de l'expérience, il y avait :

I9 sceurs totalisant 78267 coups/mn;

I6 étrangères totalisant 20 I 08 coups $/ \mathrm{mn}$;

38 nourrices totalisant 288 I 88 coups $/ \mathrm{mm}$;

Le taux de radioactivité moyenne pour une sœur est de $78267:$ I9, ce qui nous donne, une fois le calcul fait, 4 II 9 coups/mn ; pour une étrangère, il sera de 20 Io 8 : I 6 , soit I 256 coups $/ \mathrm{mn}$, et pour une nourrice, de 2888 I $8: 38$, c'est-à-dire de 7587 coups $/ \mathrm{mn}$,

Une fois établi le taux de radioactivité moyenne d'une abeille de chaque lot, nous calculons ce que représente en pourcentage le chiffre trouvé pour les sœurs et les étrangères, par rapport à celui trouvé pour les nourrices :

$$
\begin{aligned}
& \text { soit, pour les scurs : } \frac{4 \mathrm{rIg} \times \text { I00 }}{7587}=54 \% \\
& \text { pour les étrangères : } \frac{\mathrm{I} 256 \times \text { I0O }}{7587}=\mathrm{I} 6 \%
\end{aligned}
$$

Dans cette expérience, nous dirons que les scurs représentent 54 p. Ioo de la radioactivité des nourrices, tandis que les étrangères n'en représentent que i 6 p. Ioo.

Les résultats de cet essai $n^{0} 3$ sont traduits par deux points que nous réunissons : le segment SE ainsi formé fait un angle avec l'axe des ordonnées ; plus cet angle sera petit, plus la mortalité aura été similaire chez les sœurs et chez les étrangères. La projection du segment sur l'axe des ordonnées matérialise la différence $\Delta$ du taux de radioactivité des deux lots d'ouvrières (fig. $6,7,8,9$. ).

Chaque expérience sera ainsi représentée par un segment de droite. 
A) - Expérience portant sur deux jours au maximum.

Huit essais ont été faits dans ces conditions.

Le $n^{\circ}$ I et le no 8 ont montré pour les étrangères une radioactivité supérieure à celle de leurs sœurs. Les autres, au contraire, montrent une supériorité très nette chez les sœurs. Si on fait la moyenne des taux de radioactivité des abeilles nourries dans les huit essais, on obtient les chiffres suivants :

37 p. roo pour les sours ;

$25 \mathrm{p}$. Ioo pour les étrangères ;

Les différences de radioactivité entre les deux lots ne sont pas très importantes, mais il semble, d'après les résultats globaux, que les sæurs se font plus facilement nourrir que les étrangères.

Si nous regardons la pente des segments, nous remarquons que dans sept cas sur huit, ce sont les abeilles les plus radioactives qui montrent la mortalité la plus faible. Seul l'essai 4 présente une pente inversée : ceci rejoint les affirmations de KaI,IFMAN. Mais, étudions plus particulièrement les résultats donnés par les essais 7 et 8 . Dans ces cagettes, les abeilles provenaient de deux ruches que nous appellerons ruche $\mathrm{A}$ et ruche $\mathrm{B}$. Les expériences ont été faites symétriquement : en 7 la ruche A fournissait les nourrices et en 8 c'était la ruche $B$ qui les fournissait. Voici comment nous pourrions indiquer la composition des cagettes dans un schéma qui parlera plus clairement.

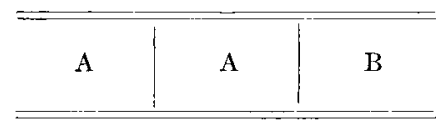

essai 7

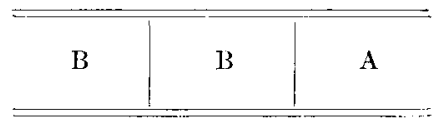

essai 8

Une des ruches présente une mortalité importante : ses abeilles utilisées comme " sœurs ) comptent 7 mortes, et employées comme étrangères elles en comptent I I : la mortalité est plus grande chez les étrangères que les chez sours.

Mais si nous considérons l'autre ruche, aucune de ses abeilles, que ce soit en tant que scur ou en tant qu'étrangère, n'est morte. Se baser sur la mortalité nous paraît donc insuffisant comme moyen de contrôle.

D'autre part, le taux de radioactivité moyen par abeille de chaque lot nous permet de constater les phénomènes suivants :

Pour 1'essai 7 , les sœurs et les étrangères sont aussi radioactives les unes que les autres et la cagette 8 , par contre, nous montre une supériorité des étrangères sur les sœurs.

\begin{tabular}{c|c|c|c}
\hline \hline & Sours & Nourrices & Étrangères \\
\hline Essai 7 & -1286 & $30 \%$ \\
\hline Essai 8 & $-29 \%$ & 2630 & $32 \%$ \\
\hline $7 \%$ & $-12 \%$ \\
\hline
\end{tabular}


Ces seuls faits nous montrent que les résultats donnés par le comptage de la radioactivité des vivantes et ceux fournis par l'étude de la mortalité sont en désaccord.
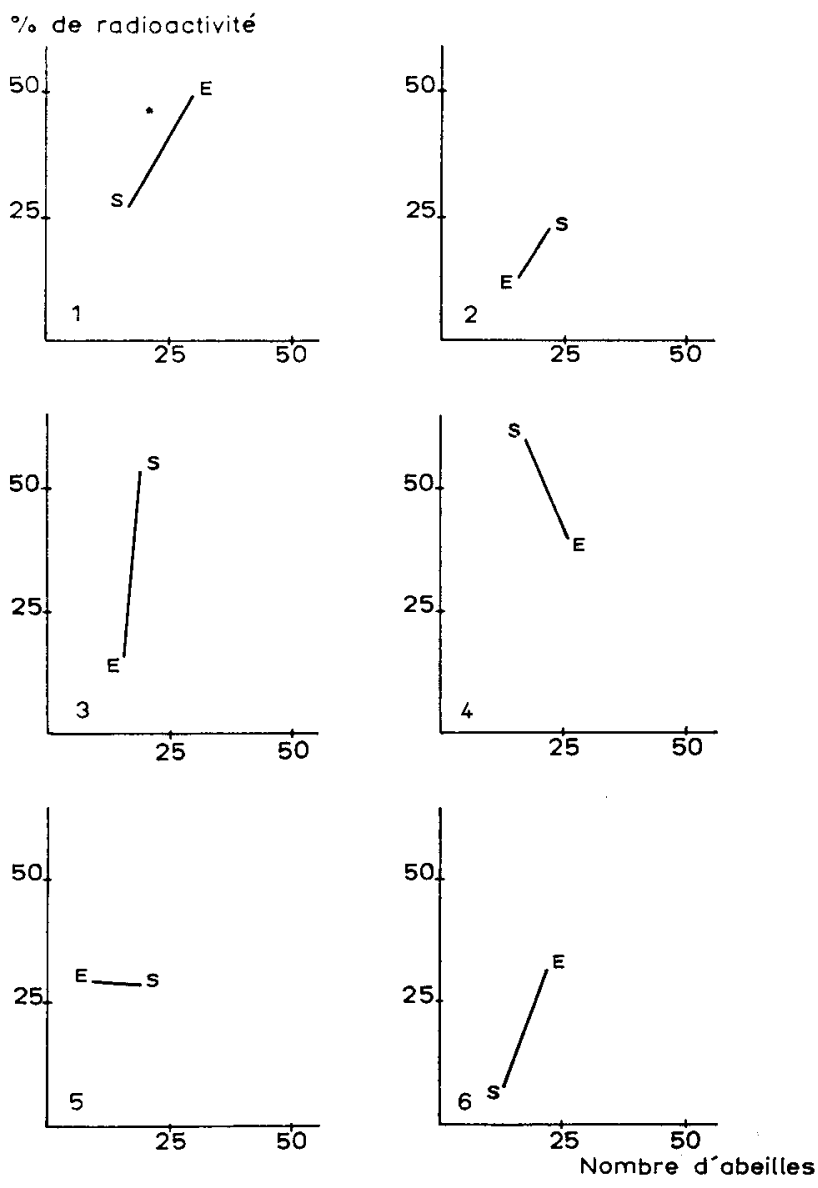

Echanges portant sur deux jours

FIG. 6. - Représentation graphique de l'intensité des échanges

$\mathrm{B}$ - Expérience portant sur quatre jours.

Nous avons répété trois fois l'expérience : essai 9, IO, II. Ce n'est évidemment pas suffisant pour établir une règle ; mais, malgré tout, nous obtenons trois résultats dans le même sens. Le pourcentage de radioactivité des sœurs établi par rapport à la moyenne de radioactivité des nourrices est de $45 \mathrm{p}$. IoO, et celui des étrangères est de $33 \mathrm{p}$. roo.

Il apparaît donc que les résultats de ces expériences vont rejoindre ceux des expériences portant sur un temps inférieur à deux jours. 
Les cagettes 9 et ro étaient jumelées, c'est-à-dire que les abeilles qui ont donné les étrangères de 9 étaient nourrices et sœurs de Io (voir schéma précédent).
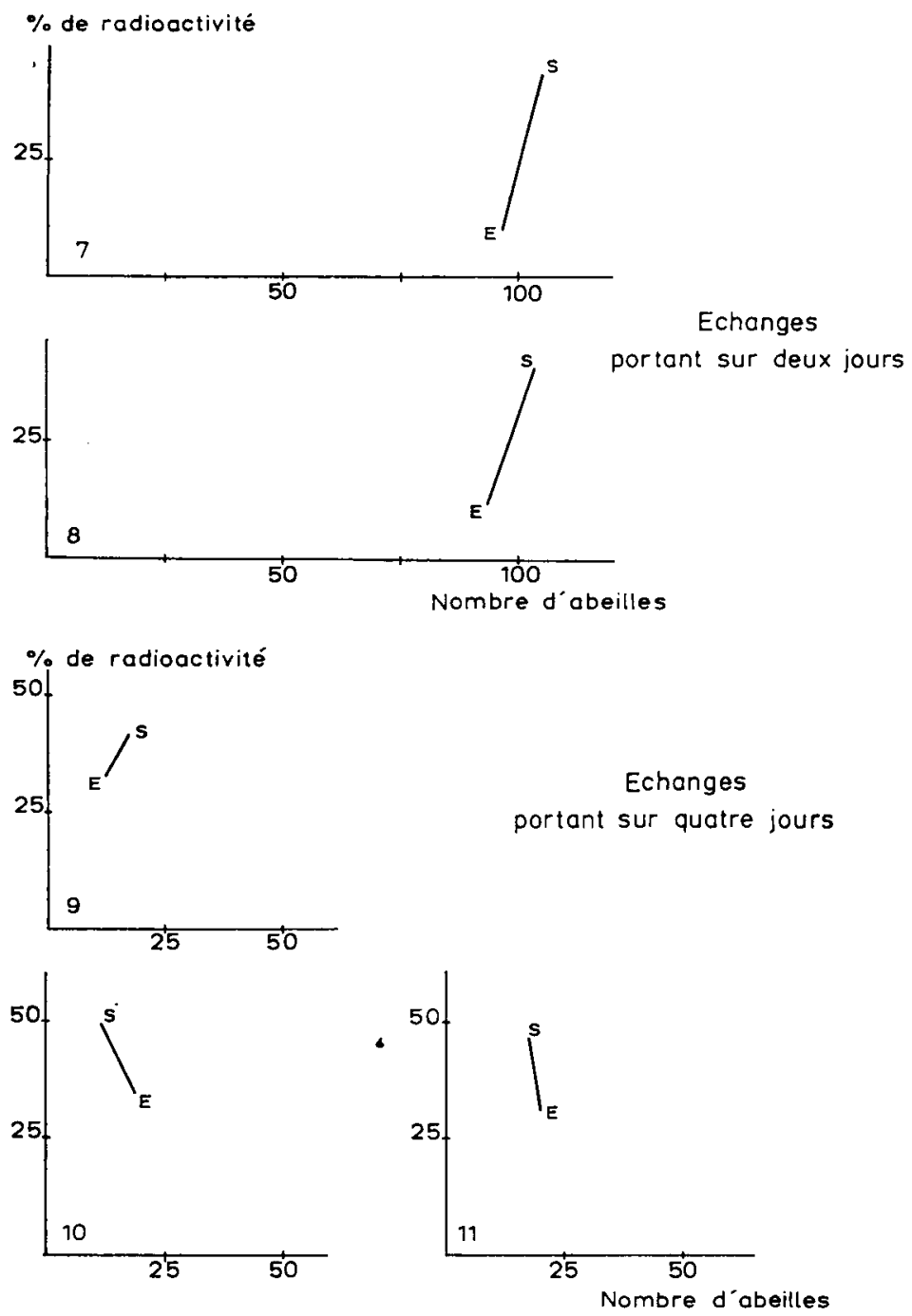

Fig. 7. - Représentation graplique de l'intensité des échanges

\section{$\mathrm{C}$ - Expérience portant sur six jours et plus.}

Les essais de cette série vont de I 2 à 22 inclus.

Seules les expériences I4 et I5 ont donné chez les sœurs un pourcentage de radioactivité supérieur à celui des étrangères. Toutes les autres, c'est-à-dire huit sur dix des expériences faites ont montré une légère supériorité de la radioactivité chez les étrangères. Il nous a semblé qu'au bout d'un certain temps, supérieur à 
quatre jours, les nourrices alimentaient plutôt les étrangères que leurs sœurs. Nous pourrons dire, semble-t-il, que les nourrices ne font plus aucune différence entre les sœurs et les étrangères.

KaLIFMAN (I955) l'avait signalé, en disant qu'au bout de trois jours il n'y avait plus de différence de mortalité entre les sœurs et les étrangères. L_EcomTE (I96I), étudiant les réactions des abeilles, vis-à-vis d'abeilles d'une ruche étrangère introduites dans leur cagette, retrouve ces phénomènes. D'après lui, le comportement est très différent lorsqu'on réunit deux groupes d'abeilles séparés par une grille, sui-
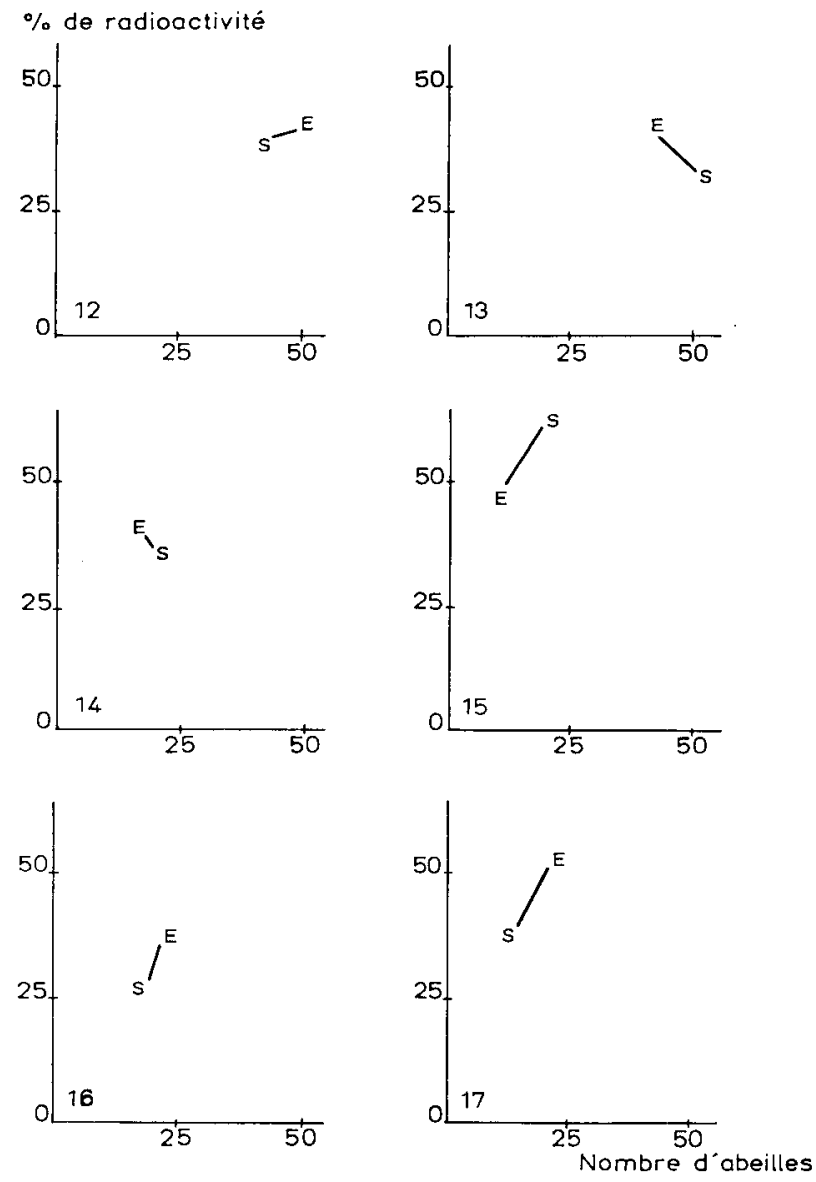

Echanges portant sur plus de six jours

FIG. 8. - Représentation graphique de l'intensité des échanges

vant que les deux populations possèdent ou non chacune leur propre réserve de nourriture. L'échange de nourriture paraît nécessaire et suffisant pour établir et entretenir entre les deux populations un lien évitant l'apparition du comportement agressif lors d'une réunion. 
Or, cela ne nous surprend pas, parce que nous savons que les abeilles d'une même ruche, nées d'une même reine, ne sont plus que demi-sœurs; en effet, $A L_{1} B E R$, JORDAN et RUTTNER (I955) ont montré qu'une reine pouvait être fécondée par huit mâles et même plus. Ce ne sont donc pas les liens parentaux qui font que les abeilles se reconnaissent comme appartenant à une même communauté, mais d'autres facteurs beaucoup plus complexes.

I a supériorité des échanges qui semble se placer du côté des étrangères ne seraitelle pas due au fait que les nourrices, moins généreuses avec elles au début, les ont obligées à demander davantage?
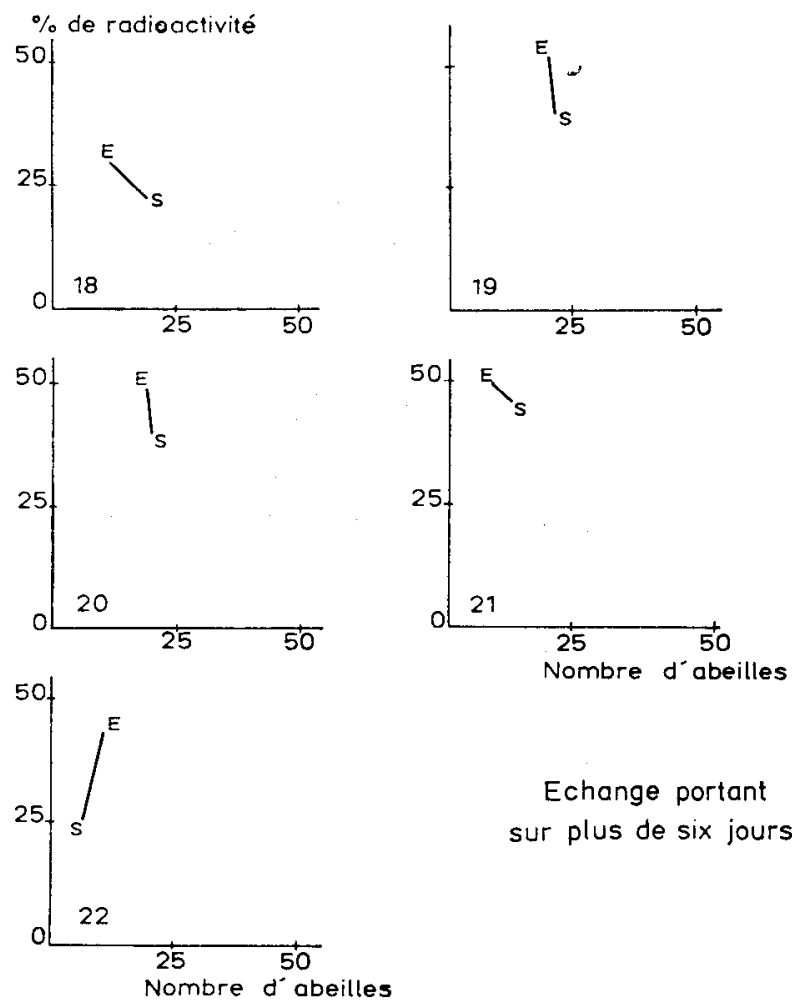

Echange portant sur plus de six jours

FIG. 9. - Représentation graphique de l'intensité des échanges

Ceci est en accord avec les travaux de FREE (I957) qui montre que la quantité de nourriture que les abeilles isolées gardent dans leur jabot est en relation avec leurs expériences antérieures. Le jabot d'une abeille que l'on a affamée, puis remise en présence de nourriture, est plus lourd que celui d'une abeille non privée de nourriture.

Les différentes séries d'expériences nous ont fourni des résultats assez difficites à interpréter. Il serait nécessaire que ces travaux soient repris à plus grande échelle, pour qu'on puisse leur appliquer des calculs statistiques. 


\section{II. - ÉCHANGE DE NOURRITURE ENTRE LES MÂLES ET LES OUVRIÈRES}

On pense généralement que les mâles sont incapables de s'alimenter seuls; OERTEL, et al. (I953) placent dans une cagette double des mâles séparés des ouvrières pat une grille. Bien que les mâles aient à leur disposition une nourriture normale, ils deviennent radioactifs quand, dans l'autre compartiment, les ouvrières sont nourries d'une substance radioactive. Ils ont donc préféré se faire alimenter à travers les grilles plutôt que de profiter de la nourriture mise à leur portée. Ces auteurs attribuent ces échanges au fait que les ouvrières leur donneraient du sucre interverti, surtout du lévulose, l'organisme des mâles n'étant pas capable, semble-t-il, de transformer le saccharose en hexoses. Cependant Gosswald et KLOFT (r958) déclarent sans donner de détails expérimentaux, que les mâles peuvent se nourrir activement et donner de la nourriture aux ouvrières.

Von Mrndt (I.96I) précise que les mâles ne reçoivent pas de sécrétion de glandes pharyngiennes, mais uniquement le contenu régurgité du jabot des ouvrières. Il souligne, de plus, qu'à l'inverse de ce qu'on voit entre ouvrières et reines, on n'observe pas de faux-bourdon qui nourrisse d'autre individu; c'est-à-dire qu'il n'y a pas de régurgitation active du jabot chez les mâles. Il dit aussi que le liquide se trouvant dans le jabot d'un mâle provient probablement inchangé du jabot de l'ouvrière.

On pouvait donc se poser les questions suivantes:

Les mâles peuvent-ils se nourrir seuls? Sont-ils capables de nourrir d'autres membres de la ruche?

$\left.I^{\circ}\right)$ Dès le début, l'avidité avec laquelle les mâles sucent le candi mis à leur disposition nous a parue frappante. Il nous a même été possible de voir deux mâles entrecroiser leur langue, peut-être pour échanger la nourriture.

\begin{tabular}{l|c}
\hline $\begin{array}{c}\text { Ouvrières } \\
\mathrm{I} \stackrel{I^{\mathrm{x}}}{ }\end{array}$ & Mâles \\
\hline $\mathrm{I}-\mathrm{I}^{\mathrm{x}}$ nourriture radioactive \\
\hline
\end{tabular}

Nous avons travaillé sur une cagette double dont les deux compartiments sont sóparés par une grille. Dans le compartiment des mâles, il n'existe aucune nourriture, par contre une nourriture radioactive, sous forme de candi, est mise à la disposition des ouvrières. Ces expériences ont porté sur vingt-quatre heures : au terme de ce délai, les animaux sacrifiés sont introduits sous le compteur.

Le comptage des survivants a donné les résultats ci-après.

L'énorme mortalité des mâles est la première constation que l'on peut faire.

Étant donné l'importance de la mortalité, nous pouvons seulement penser d'une part que les échanges ont été le fait d'un petit nombre, d'autre part que les ouvrières ne restent pas insensibles à la présence des mâles, puisqu'elles ont communiqué à quelques-uns d'entre eux une certaine radioactivité. 


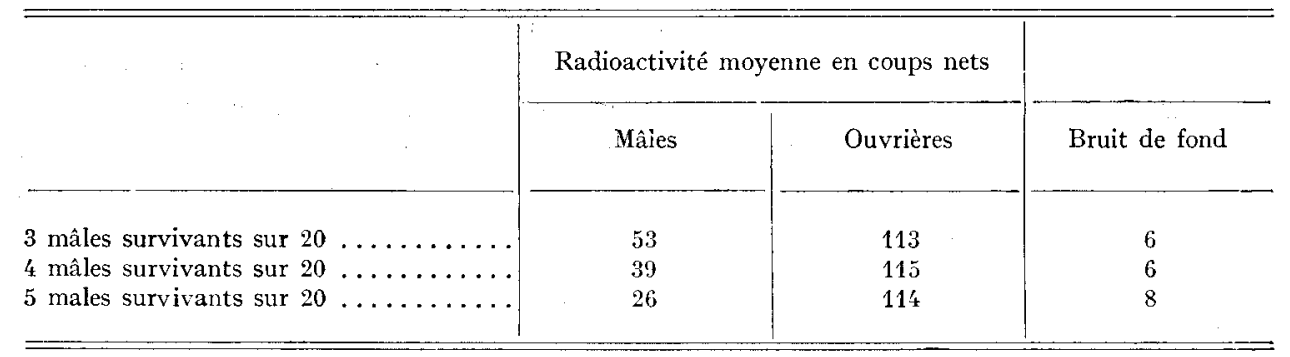

$2^{\circ}$ ) On pouvait se demander si les mâles présenteraient la même mortalité si 1 'on introduisait de leur côté une nourriture normale.

Plan d'expérience :

Plan d'expérience
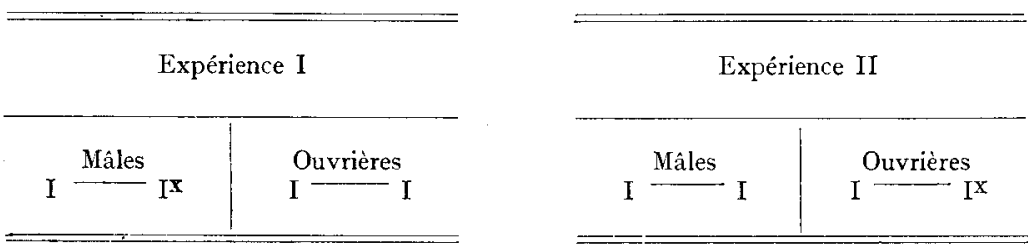

TABI.EAU I

Radioactivité exprimée en coups nets

\begin{tabular}{|c|c|c|c|c|}
\hline \multirow{3}{*}{ Bruit de fond } & \multicolumn{4}{|c|}{ Expérience II } \\
\hline & \multicolumn{2}{|c|}{ Mâles avec nourriture normale } & \multicolumn{2}{|c|}{ Ouvrières avec nourriture radioactive } \\
\hline & Moyenne & Extrêmes & Moyenne & Extrêmes \\
\hline 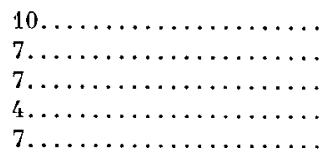 & $\begin{array}{r}281 \\
41 \\
40 \\
1 \\
13\end{array}$ & $\begin{array}{l}0-936 \\
0-296 \\
0-252 \\
0-8 \\
0-32\end{array}$ & $\begin{array}{r}982 \\
1057 \\
362 \\
123 \\
82\end{array}$ & $\begin{array}{c}10-2109 \\
245-2023 \\
242-481 \\
1-251 \\
7-286\end{array}$ \\
\hline
\end{tabular}

Nous avons mené de pair l'expérience et la contre-expérience. Les cagettes ont été organisées de la façon suivante : du candi était mis à la disposition des insectes dans tous les compartiments. Mais, tantôt le candi était radioactif dans le compartiment des mâles, tantôt dans celui des ouvrières. Ceci devait nous permettre de voir si les mâles en présence de nourriture étaient capables de se nourrir eux-mêmes, ou s'ils préféraient demander leur nourriture aux ouvrières. 
Nous avons groupé les résultats en un tableau.

I1 nous est facile de voir que, même en présence de nourriture, les mâles se font nourrir par les ouvrières, puisqu'ils deviennent radioactifs. Cette expérience confirme ce que nous montrait l'expérience précédente. La mortalité moins grande peut donner à penser que les mâles prennent aussi la nourriture qui leur est proposée dans leur compartiment en plus de ce que leur communiquent les ouvrières.

TABI,EAU 2

Radioactivité exprimée en coups nets

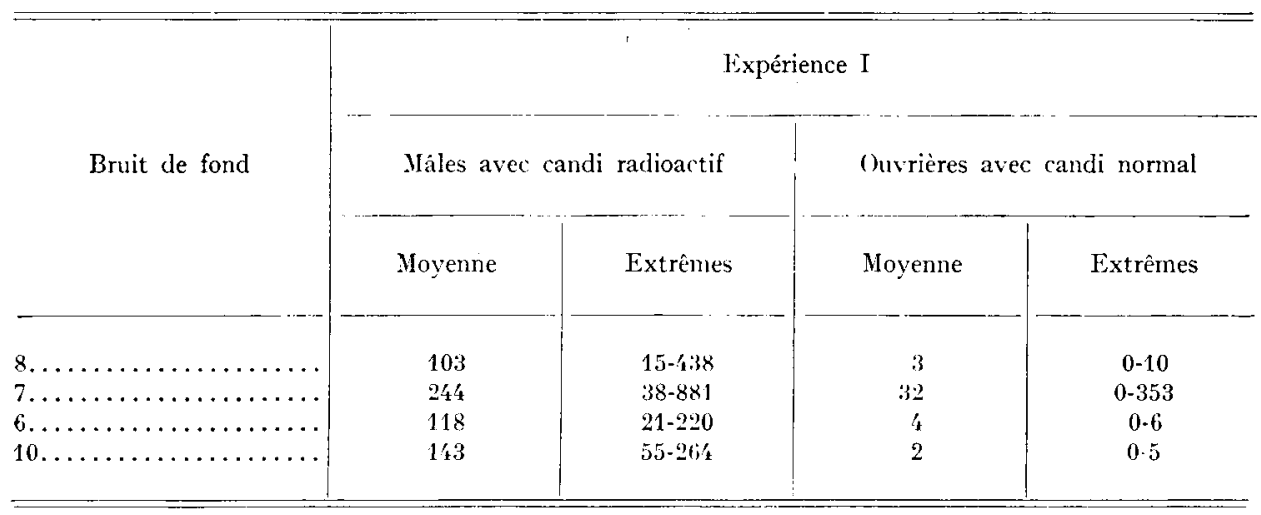

Ceci confirme les vues déjà exprimées : si les mâles sont radioactifs, cela ne peut être dû qu'à l'ingestion de la nourriture placée de leur côté, et le fait que les ouvrières deviennent légèrement radioactives indique que les mâles leur ont communiqué leur radioactivité.

Nous pouvons dire, pour conclure, que les mâles sont capables de se nourrir seuls, mais pourtant, en présence d'ouvrières, ils préfèrent se faire nourrir par elles, ces dernières recevant en retour une substance.

$3^{\circ}$ ) - Dans un nouveau type d'expérience, nous avons séparé un certain nombre de mâles et d'ouvrières d'une même ruche en deux groupes égaux de vingt mâles et de vingt ouvrières chacun. Nous mettons chaque lot dans une cagette l'un avec une nourriture radioactive, l'autre avec une nourriture normale. Au bout de deux jours, nous dissocions ces deux groupes pour en constituer deux autres :

TABI,EAU 3

Radioactivité exprimée en coups nets

\begin{tabular}{|c|c|c|c|c|}
\hline \multirow{2}{*}{ Bruit de fond } & \multicolumn{2}{|c|}{ Mâles normaux } & \multicolumn{2}{|c|}{ Ouvrières rendues radioactives } \\
\hline & Moyenne & Extrêmes & Moyenne & Extrêmes \\
\hline $8 \ldots \ldots \ldots \ldots \ldots \ldots \ldots$ & 4 & $0-34$ & $1 ' 8$ & $92-355$ \\
\hline
\end{tabular}


TABLEAU 3 (suite)

Radioactivité exprimée en coups nets

\begin{tabular}{|c|c|c|c|c|}
\hline \multirow{2}{*}{ Bruit de fond } & \multicolumn{2}{|c|}{ Mâles rendus radioactifs } & \multicolumn{2}{|c|}{ Ou vrières normales } \\
\hline & Moyenne & Extrêmes. & Moyenne & Eixtrêmes \\
\hline $9 \ldots \ldots \ldots \ldots$ & 35 & $13-137$ & 2 & $0-11$ \\
\hline
\end{tabular}

- les mâles radioactifs sont placés avec leurs sœurs non radioactives.

- les ouvrières radioactives sont placées avec leurs frères non radioactifs.

Dans chaque nouvelle cagette, nous mettons alors une nourriture normale. Au bout de deux jours, l'ensemble est sacrifié et compté dans le château de plomb.

$a$ - Cette expérience montre tout d'abord que des mâles et des ouvrières mis ensemble dans une cagette avec du candi radioactif, présentent un taux de radioactivité très différent. La moyenne par mâle est de 35 coups par minute, alors que celle des ouvrières est de I48. Ceci semble indiquer que les mâles mangent moins que les ouvrières.

$b$ - d'autre part, dans un cas comme dans l'autre, on peut voir qu'il y a eu transfert de radioactivité, bien qu'il soit plus faible des mâles vers les ouvrières, que des ouvrières vers les mâles.

$4^{\circ}$ ) - Nous avons étudié ce qui se passe dans un groupe d'abeilles lorsqu'on $\mathrm{y}$ introduit un ou deux mâles radioactifs.

Nous avons introduit dans une cagette où se trouvait une vingtaine d'ouvrières avec de la nourriture normale, des mâles radioactifs. Nous avons fait des comptages au bout de quarante-huit heures; voici le taux de radioactivité obtenu pour les ouvrières les plus radioactives :

TABLEAU 4

Radioactivité exprimée en coups nets

\begin{tabular}{|c|c|c|}
\hline Bruit de fond & Ouvrières & Mâles \\
\hline $9 \ldots \ldots \ldots \ldots \ldots \ldots \ldots$ & $32-12-13$ & 546 \\
\hline $9 \ldots \ldots \ldots \ldots \ldots \ldots$ & $8-11$ & 482 \\
\hline $9 \ldots \ldots \ldots+\ldots, \ldots, \ldots, \ldots, \ldots$ & $6-7$ & 300 \\
\hline $10 \ldots \ldots \ldots \ldots$ & $5-5$ & 548 \\
\hline
\end{tabular}


L,e mâle, caractérisé au compteur par 546 coups par minute, a très nettement transmis une substance radioactive à trois ouvrières.

Nous avons répété plusieurs fois ces expériences : dans cinq cas sur sept, nous avons eu de façon évidente contamination.

Nous avons mis ensuite des ouvrières âgées pendant vingt-quatre heures en contact par groupes de cinq avec un ou deux mâles radioactifs. Quelle que soit l'importance de la radioactivité des donneurs, les abeilles ne présentaient pas de taux de comptage très élevés; pourtant, il était indéniable qu'il y avait nettement transmission de produits radioactifs, que ce soit transmission de nourriture ou contamination par contact.

La même expérience a été faite avec des abeilles naissantes. Elle a donné les résultats suivants :

TABLEAU 5

Radioactivité exprimée en coups nets

\begin{tabular}{|c|c|c|c|}
\hline Bruit de fond & Mâles & Ouvrières & Nourriture \\
\hline 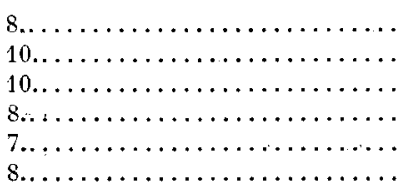 & $\begin{array}{c}1248-8096 \\
4247 \\
4524 \\
6013 \\
7805 \\
7179\end{array}$ & $\begin{array}{l}10,14,6,8,7 \\
10,13,8 \\
8,15 \\
109,46,51 \\
16 \\
108,55,258,83\end{array}$ & $\begin{array}{r}373 \\
12 \\
39 \\
371 \\
141 \\
83\end{array}$ \\
\hline
\end{tabular}

Les essais ont été faits avec seulement dix ouvrières.

Les ouvrières placées avec certains mâles, par exemple ceux caractérisés au compteur par 6 or 3 et 7 I 79 coups nets par minutes, ont montré un taux important de radioactivité : pour le premier des chiffres comme Iog,46 et $5 \mathrm{I}$, pour le second Io8, 258, 83. Il paraît étonnant que les mâles soient capables de transmettre pareille quantité de matière radioactive à plusieurs abeilles. Il est probable que les abeilles ont été contaminées d'une autre manière. C'est alors que l'examen de la nourriture nous a montré qu'elle était devenue radioactive. Le taux de radioactivité de ces abeilles n'était pas dû à l'intensité des échanges, mais au candi ingéré.

Le problème restait entier : comment la nourriture devenait-elle radioactive?

Une observation du comportement des mâles mis en cagette a apporté la solution. Il n'est pas rare de les voir régurgiter sur la nourriture. Ce phénomène a pu être observé plusieurs fois et la contamination de la nourriture en apporte une seconde preuve.

Une observation plus poussée a permis de constater que les ouvrières, loin de repousser cette nourriture polluée, la sucent avec avidité. Nous ne saurions dire si ces phénomènes sont généraux ou s'ils sont liés aux conditions de l'expérience Ce phénomène s'est reproduit en introduisant des mâles radioactifs dans des cagettes contenant d'autres mâles non radioactifs. La nourriture devenait alors radioactive.

Par exemple : deux mâles (5539 et 4564), (nous donnons entre parenthèses leur radioactivité en coups nets), ont rendu deux autres mâles légèrement radioactifs (6 et 6 ), tandis que, pour la nourriture, le compteur enregistre 25 coups. Du fait 
que la nourriture présente un taux de radioactivité plus élevé que les mâles contaminés, on peut déduite que cette contamination se fait autant par la pollution de la nourriture que par l'échange de nourriture. La même chose pour deux mâles (7028 et 6877) qui donnent I9, I7, 23, 54 chez les mâles, la nourriture faisant $9 \mathrm{I}$.

$5^{\circ}$ ) - Pour éviter que les insectes non radioactifs le deviennent en mangeant une nourriture polluée, nous avons retiré le candi au moment où nous introduisions les animaux radioactifs. L'expérience ne dure pas plus de $6 \mathrm{~h}$.

Deux mâles (I073 et I I 47) transmettent à des mâles les taux de radioactivité de $28,8,9,9,8,8$.

Deux mâles (686 et 692) transmetteñt à un seul mâle radioactif faisant I4 coups par minute.

Le comptage des différentes parties du corps des frères devenus radioactifs montre que les pattes sont peu radioactives; par contre, l'abdomen l'est plus et on peut penser que leur radioactivité vient de ce qu'ils ont ingurgité.

$6^{\circ}$ ) - Dans une dernière série d'expériences, nous a vons voulu savoir si les mâles étaient capables de nourrir les ouvrières.

Dans une cagette double, nous avons mis la nourriture seulement du côté où se trouvaient les mâles; au bout de quarante-huit heures, nous avons pu constater que les ouvrières étaient devenues radioactives. Nous avons pu voir, d'autre part, un mâle et une ouvrière entrecroiser leur langue.

Nous avons aussi remarqué sur les ouvrières allongeaient démesurément leur langue, de telle façon qu'elles arrivaient à lécher la grille du côté des mâles. Fìn frottant cette grille avec un coton que nous avons passé sous le compteur, nous avons pu constater qu'elle était fortement radioactive. Dans ces conditions, il est impossible de dire si la radioactivité des abeilles était due à un échange avec les mâles ou au fait qu'elles aient elles-mêmes léché la grille.

Malgré tout, bien que cela soit du domaine des hypothèses, rien n'empêche de penser que cette régurgitation ne soit une réponse des mâles à la sollicitation des ouvrières. La régurgitation des mâles pourrait bien être dans la ruche un phénomène normal. Et nous avons vu que les ouvrières paraissaient attirées par ces régurgitations.

\section{III. - Échange de NOURRITURE ENTRE REINES ET OUVRIÈRES}

La reine est toujours entourée d'un certain nombre d'abeilles. L'attraction qu'exerce la reine sur son entourage se traduit par une " cour " d'ouvrières se tenant le plus près d'elle possible et lui présentant souvent de la nourriture. On peut voir les abeilles de la " cour » lécher avec persistance les différentes parties du corps de la reine.

PaIs J. (I96I) explique comment, en léchant, la reine, les ouvrières absorbent une substance inhibitrice du développement ovarien et de la construction des cellules royales.

Les rapports qui existent entre la reine et les ouvrières sont mal connus. Rendre une reine radioactive permettrait peut-être de mieux connaître les échanges de nourriture.

Nous avons laissé la reine se nourrir elle-même. Pour cela le phosphore 32 lui 


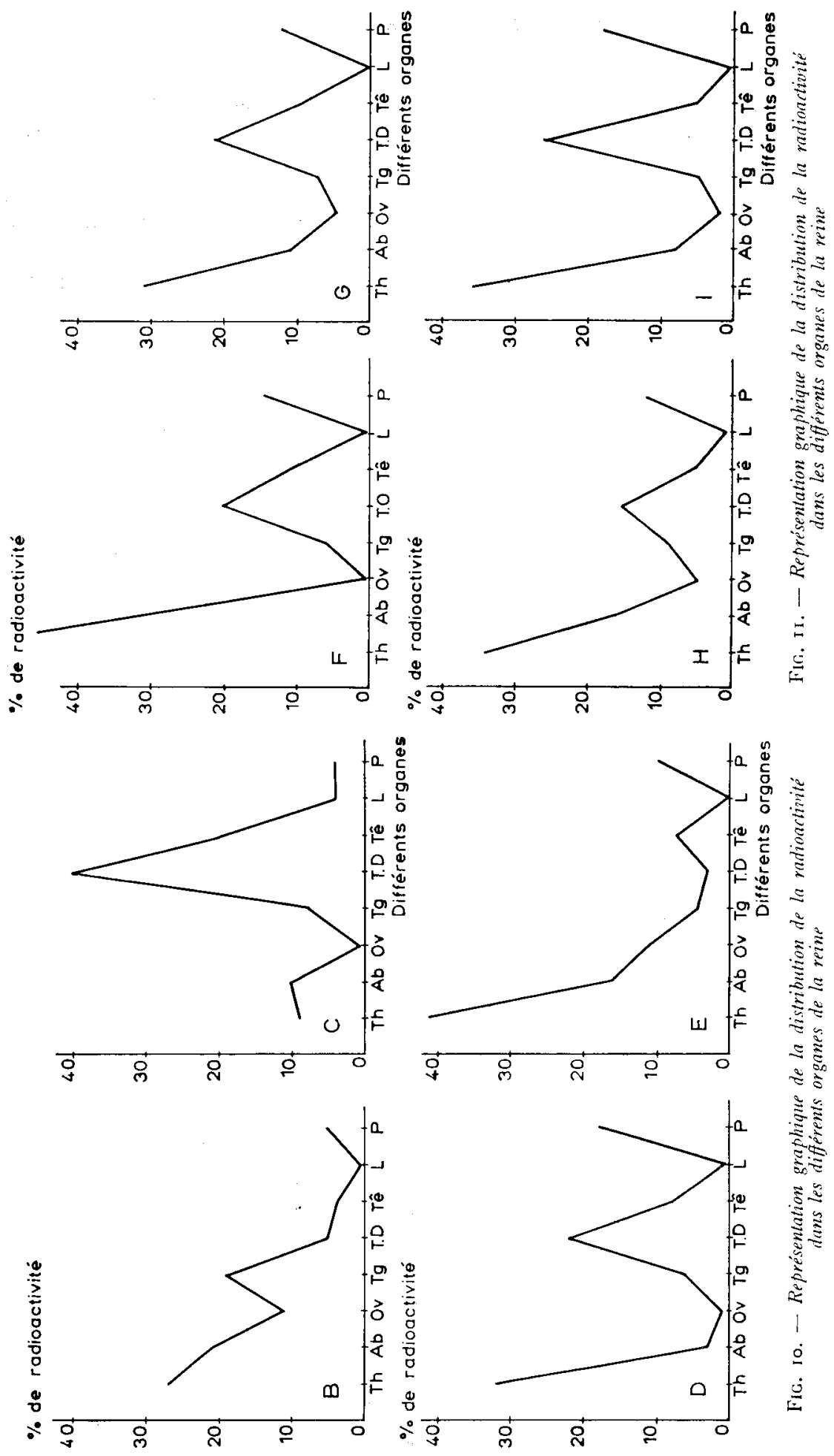


a été donné mélangé à du miel dans un nourrisseur constitué d'un tube de verre dont le rayon est assez petit pour permettre à la reine d'introduire uniquement la tête.

Les reines supportent mal l'absence d'ouvrières, plusieurs sont mortes au cours de cet isolement, mais toutes ont effectivement mangé. La simple observation nous montre qu'elles peuvent se nourrir. Une preuve supplémentaire en est donnée par la dissection des cadavres de reines mortes dans ces conditions. Les figures ro et II nous montrent le taux de radioactivité des différents organes. Les reines C. D. G. H. I. comptées à leur mort, ont un tube digestif dont le taux de radioactivité varie entre I 5 et $40 \mathrm{p}$. Ioo par rapport à la radioactivité totale de ces reines.

Le comptage d'un organe ayant une grande surface, comme les ailes, n'a rien donné de positif sous le compteur. Nous avons pensé alors que les reines étaient devenues radioactives sans être polluées extérieurement.

\section{$\mathrm{I}^{\mathrm{O}}$ - Même lot d'ouvrières mis en contact plusieurs jours avec une reine radioactive}

Les reines radioactives (nous leur avons attribué une lettre pour faciliter l'exposition des résultats), ont été placées dans une cagette normalement équipée en présence d'un lot de vingt ouvrières naissantes. Au début, la radioactivité des ouvrières était mesurée chaque jour. Elles étaient ensuite placées à nouveau en présence de la reine. Les abeilles sont introduites dans le château de plomb dans une petite boîte en carton. Pour pouvoir les reconnaître d'un jour à 1'autre, les abeilles étaient marquées à la peinture.

Figurent uniquement sur le tableau les abeilles dont le taux de radioactivité est supérieur au double du bruit de fond. Tous les chiffres sont indiqués en coups nets.

D'après ces chiffres, il apparaît assez nettement que les abeilles mises en contact avec la reine sont devenues radioactives, et ce phénomène est encore plus net le second jour.

TABLEAU 6

Reine $A-620$ coups par minute

Radioactivité exprimée en coups nets

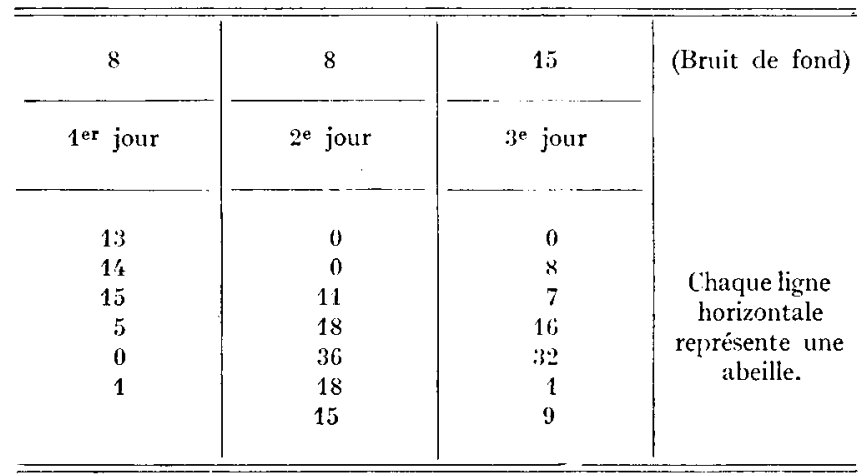


TABLEAU 7

Reine $B-720$ coups par minute

Radioactivité exprimée en coups nets

\begin{tabular}{c|c|c|c}
\hline 7 & 8 & 15 \\
\hline $1^{\text {er }}$ jour & $2^{\text {e jour }}$ & (Bruit de fond) \\
\hline 15 & $3^{\text {e jour }}$ & \\
\hline 5 & 17 & 22 \\
19 & 12 \\
\hline
\end{tabular}

Ici, les échanges auraient été le fait de deux abeilles set1lement. Ces deux expériences ont été faites avec des reines relativement peu radioactives. Rendre les reines plus radioactives devait nous permettre de déceler plus facilement les échanges.

\section{TABLEAU 8}

Reine $B-17$ zoo coups par minute

Radioactivité exprimée en coups nets

\begin{tabular}{|c|c|c|}
\hline 12 & 10 & (Bruit de fond) \\
\hline $1^{\text {er jour }}$ & $2^{e}$ jour & \\
\hline 177 & 183 & \\
\hline 438 & $4 \mathbf{4} 0$ & \\
\hline 103 & 212 & \\
\hline 230 & 236 & \\
\hline 139 & 178 & \\
\hline 26 & 151 & \\
\hline 88 & 136 & \\
\hline 38 & 91 & \\
\hline 80 & $12^{\prime}$ & $\begin{array}{l}\text { Moyenne des } \\
\text { vingt abeilles }\end{array}$ \\
\hline
\end{tabular}

Nous voyons que les abeilles radioactives le restent le second jour avec, pour certaines, un accroissement notable du taux de radioactivité, en particulier pour celles qui passent de IO3 à 2 I2 et de 26 à I5I.

Si nous considérons la moyenne de radioactivité par abeille, nous voyons qu'elle passe de 80 coups par minute le premier jour, à I 24 coups par minute le second. Cet accroissement important suppose que cet échange n'est pas le fait du hasard avec l'une ou l'autre abeille, mais qu'il y a communication de la radioactivité de la reine aux ouvrières. Cette redistribution est très inégale puisqu'une abeille arrive à un 
taux de radioactivité de 440 coups par minute, tandis que pour la moins radioactive nous comptons seulement 25 coups par minute.

Reine $A-6940$ coups par minute.

Au cours de l'expérience avec cette reine, une certaine quantité d'abeilles a pu s'échapper de la cagette où elles étaient enfermées. Nous avons dûr renouveler entièrement le lot d'abeilles naissantes le second jour. C'est alors que nous avons obtenu les résultats suivants :

\section{TABLEAU 9}

Reine $A-6940$ coups par minute Radioactivité exprimée en coups nets

\begin{tabular}{|c|c|c|c|}
\hline 9 & 10 & 8 & \\
\hline $\begin{array}{c}1^{\text {er }} \text { lot } \\
1^{\text {er }} \text { jour }\end{array}$ & $\begin{array}{c}2^{\mathrm{e}} \text { lot } \\
2^{\mathrm{e}} \text { jour }\end{array}$ & $3^{e}$ jour & (Bruit de fond) \\
\hline $\begin{array}{l}21 \\
25 \\
67 \\
65\end{array}$ & $\begin{array}{r}33 \\
86 \\
0\end{array}$ & $\begin{array}{l}34 \\
69 \\
30\end{array}$ & \\
\hline 39 & 9 & 1't & \\
\hline
\end{tabular}

Dans le second lot d'abeilles placé avec la reine $\mathrm{A}$, il est intéressant de constater que la reine n'avait pas reçu de nourriture radioactive depuis quarante-huit heures. Or, au bout de ce temps, on peut remarquer que les ouvrières vivant avec elle sont devenues légèrement radioactives; de plus, la radioactivité moyenne de l'ensemble des ouvrières du second lot s'est accrue entre le second et le troisième jour. Cette constatation nous a donné à penser que nous obtiendrions peut-être des résultats plus probants en renouvelant chaque lour le lot d'abeilles mises en précence de la reine.

\section{$2^{\circ}$ - Lot d'ouvrières changé après chaque comptage.}

Le fait de renouveler le lot d'abeilles après chaque comptage devait nous permettre de déceler le moindre échange sans être gênés par la trophallaxie qui règne entre les ouvrières. Cette technique évite de marquer les abeilles à la peinture. De plus, nous n'avions pas à les placer dans des petites boîtes, ce qui permet un meilleur comptage. Fin outre, les abeilles mises en présence de la reine, étaient dans un état physiologique parfait.

Il n'est pas impossible de penser que les premières abeilles mises avec la reine acquièrent une certaine radioactivité en léchant le corps de la reine et en suçant le miel resté sur la langue. Par contre, il nous a semblé possible d'estimer qu'une fois la " toilette " de la reine faite, le second lot d'abeilles ne peut devenir radioactif que par un échange de nourriture. 
Reine E. -4460 coups le jour de la mise en expérience

4400 coups le $2^{\mathrm{e}}$ jour

3240 coups le $4^{\mathrm{e}}$ jour

$279^{\circ}$ coups le $6^{\mathrm{e}}$ jour

Radioactivité exprimée en coups nets

\begin{tabular}{|c|c|c|c|}
\hline 9 & 7 & 11 & (Bruit de fond) \\
\hline $\begin{array}{l}1^{\text {er }} \text { lot } \\
\text { ye jour }\end{array}$ & 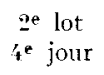 & $\begin{array}{c}3^{e} \text { lot } \\
6^{\mathbf{e}} \text { jour }\end{array}$ & \\
\hline $\begin{array}{l}21 \\
10 \\
16 \\
32 \\
16\end{array}$ & $\begin{array}{l}20 \\
34 \\
13 \\
25 \\
16\end{array}$ & $\begin{array}{l}20 \\
38 \\
18 \\
39 \\
33^{\prime} \\
17\end{array}$ & \\
\hline 7 & 9 & 10 & $\begin{array}{c}\text { Moyenne des } \\
\text { abeilles. }\end{array}$ \\
\hline
\end{tabular}

C'est dans cet esprit que nous avons entrepris les expériences suivantes:

La reine est nourrie avec du miel additionné de phosphore 32 pendant vingtquatre heures et ensuite remise dans une cagette propre dans laquelle on renouvelle les ouvrières tous les deux jours. Les ouvrières de chaque lot sont tuées après leur séjour avec la reine.

On s'efforce de mettre avec la reine des lots d'ouvrières naissantes provenant du même cadre de couvain.

Les abeilles ont été changées toutes les quarante-huit heures. La reine $\mathrm{E}$ a été laissée pendant trois semaines avec des ouvrières; nous les avons comptées au bout de ce temps-là. Pour les plus radioactives de ce quatrième lot, nous avons pu compter 8,8 , Io, 8 coups nets pour un bruit de fond de Io; la reine était elle-même passée de 2790 à 792 coups par minute.

Il nous semble donc indéniable qu'il existe des échanges de la reine vers les ouvrières.

Cette expérience met en évidence les faits suivants :

Le premier lot est nettement radioactif, puisque la moyenne des ouvrières est de 25 coups par minute; le second lot, au contraire, l'est très peu, sa moyenne est de 4 ; le troisième lot, par contre, nous donne le chiffre de $\mathrm{I} 4$. I1 nous semble possible de dire que la reine communique par voie buccale la radioactivité qu'elle a reçue, aux ouvrières qui sont en sa présence.

D'autres résultats ont été obtenus, il nous a semblé fastidieux de les analyser un à un ; il suffit de signaler qu'ils sont en accord avec ceux que nous venons de citer. 
TABLEAU I I

Reine $F$. I $99 \mathrm{I}$ coups par minute Radioactivité exprimée en coups nets

\begin{tabular}{|c|c|c|c|}
\hline $\begin{array}{l}\text { 1er lot } \\
2 \mathrm{e} \text { jour }\end{array}$ & $\begin{array}{l}2^{e} \text { lot } \\
4^{\mathrm{e}} \text { jour }\end{array}$ & $\begin{array}{c}3^{e} \text { lot } \\
6^{e} \text { jour }\end{array}$ & \\
\hline 9 & 10 & 8 & (Bruit de fond) \\
\hline 29 & 15 & 12 & \\
\hline 9 & 8 & 18 & \\
\hline 16 & 8 & 22 & \\
\hline 14 & 8 & 64 & \\
\hline 41 & 9 & 32 & \\
\hline 32 & 6 & 22 & \\
\hline 253 & & 16 & \\
\hline 25 & 4 & 1 't & $\begin{array}{l}\text { Moyenne des } \\
\text { abeilles. }\end{array}$ \\
\hline 1991 & 1351 & 992 & Reine $\mathrm{H}$ \\
\hline
\end{tabular}

Il serait intéressant de savoir où se localise, chez la reine, la radioactivité.

La dissection des cadavres de reines donne des résultats difficiles à interpréter.

Nous avons, d'autre part, tracé la courbe du pourcentage de radioactivité des différents organes provenant de la dissection des reines, en les plaçant dans un ordre arbitrairement choisi, mais toujours le même (fig Io et II). Il est frappant de constater que plusieurs des courbes présentent la même physionomie. faut Mais, il préciser que, bien que la radioactivité semble se localiser dans les mêmes organes, les reines sont mortes dans des circonstances différentes. Certaines sont mortes au cours de leur nourrissement, d'autres quelques jours après, certaines quelques mois après ; d'autres encore après avoir été nourries plusieurs fois avec du phosphore 32 . Les reines constituent un matériel rare et onéreux, c'est pourquoi il serait plus intéressant d'attaquer cette question en utilisant la technique des auto-radiographes.

Quoi qu'il en soit, de toutes cesexpériences se dégage une constatation indéniable: une reine communique une certaine quantité de radioactivité aux ouvrières en présence desquelles elle a été mise, mais il est certain que le taux de radioactivité acquis de la reine par les ouvrières est très taible par rapport à celui de la reine.

Il serait intéressant de pouvoir préciser la quantité et la nature des échanges ainsi décelés.

\section{CONCLUSION}

L'utilisation des radio-isotopes en éthologie des insectes sociaux est relativement neuve et si, en ce qui concerne les Fourmis, les recherches de GösswaLD et de son école ont déjà atteint un certain développement, il n'en est pas de même en ce qui concerne les Abeilles qui avaient été fort peu étudiées sous ce jour. 
Après la mise au point des techniques, il me fut donné de pouvoir apporter une réponse, au moins provisoire, à trois grandes questions :

Io Les ouvrières préfèrent-elles donner de la nourriture ou en demander à leurs scurs?

$2^{\circ}$ Les mâles peuvent-ils se nourrir seuls et peuvent-ils nourrir les ouvrières?

$3^{\circ}$ Les ouvrières reçoivent-elles quelque chose des reines?

\section{Échanges entre ouvrières}

Les résultats assez contradictoires que nous avons obtenus montrent qu'on ne peut accepter comme règle générale les conclusions de KaLIFMAN J. (I955) qui laissent supposer que, dans dans tous les cas, les échanges de nourriture entre ouvrières de même ruche s'effectuent plus aisément qu'entre des ouvrières appartenant à des ruches différentes. L'hétérogénéité des résultats laisse supposer l'existence d'un facteur qui nous échappe, soit qu'il provienne des conditions expérimentales, soit que les différentes ruches réagissent de manière différente

\section{Échanges entre mâles et ouvrières}

Nous avons montré sans ambiguïté que les mâles sont capables, dans une certaine mesure, de se nourrir seuls, mais qu'en présence d'ouvrières ils préfèrent se faire nourrir par ces dernières. D'autre part, il y a transfert de radioactivité des mâles vers les ouvrières, ẹe transfert pouvant être dî, dans certains cas, à l'absorption par les ouvrières des régurgitations des mâles déposées sur le substrat, dans d'autres cas, à un échange direct de nourriture, que nous avons d'ailleurs pu observer.

Les faux-bourdons sont certainement les habitants de la ruche les moins bien connus, aussi faut-il s'attendre à ce que des recherches à ce sujet apportent des résultats nouveaux. En particulier, les échanges de nourriture dans le sens inverse de celui qui était couramment admis, c'est-ì-dire vers les ouvrières, n'avait pas été signalé à notre connaissance. De même, il serait très intéressant d'étudier si dans le cadre, non plus d'une cagette expérimentale, mais d'une colonie normale, les régurgitions de mâles existent et si elles jouent un rôle important.

\section{Échanges entre reines et ouvrières}

L'existence d'un transfert de radioactivité des reines aux ouvrières paraît maintenant une chose certaine. Quand on connaît le rôle important que joue la reine dans la colonie par les substances qu'elle sécrète, on conçoit l'intérêt primordial qu'offre la possibilité d'avoir des données numériques sur l'importance des échanges par voie buccale.

D'autre part, on sait que dans la ruche un petit nombre d'abeilles formant ce qu'on nomme " la cour ", entoure la reine et participe directement à ces échanges. Il semble en être de même dans les colonies expérimentales très restreinte (20 ouvrières) et les échanges directs avec la reine ne sont que le fait d'un très petit nombre, deux parfois, d'individus. Une cagette expérimentale peut donc être considérée à cet égard comme une colonie en réduction avec une organisation sociologique similaire. 
Ceci est à rapprocher des résultats de Roubaud E. (I950) qui trouve une division dans de très petites colonies et de ceux de LECOMTE J. (I G6I) qui, en ce qui concerne 1'agressivité, constate dans des cagettes expérimentales l'existence d'une spécialisation. L'utilisation des tracteurs radioactifs dans le but de mieux connaître la structure fine de la société d'abeilles est certainement une méthode très féconde. Dans ce travail, nous n'avons pu qu'effleurer les principales questions qui peuvent venir à l'esprit quand on s'intéresse à ces problèmes. $\mathrm{Il}$ est à souhaiter que ces recherches se poursuivent dans l'une ou l'autre des directions que j'ai étudiées et dans bien d'autres encore.

Reçu pour publication en janvier 1963 .

\section{SUMMARY}

A RADIO-ISOTOPE STUDY OF THE FOOD EXCHANGE BETWEEN QUEENS, MALES AND WORKERS OF Apis mellifica L.

The object of this study was to introduce the use of radioactive tracers into the examination of the exchange of food between the different classes of inhabitants of the hive.

The optimum experimental conditions were first defined. The radioisotope used was phosphorus-32 which was incorporated into the food.

During the first 48 hours there was an indication that the nurse bees showed some preference for their sisters. Later on contradictory evidence was obtained.

Although preferring to be fed by workers, the males appeared, under certains conditions, to be able to feed themselves.

The workers received their food trom the males either by the ingestion of regurgitated material, or by direct feeding.

The queens passed food or substances containing radio-active tracers to the workers; in one restricted group certain privileged workers participated to a greater extent than the others in these exchanges.

\section{RÉFÉRENCES BIBLIOGRAPHIQUES}

Alber M. A., Jordan R., Ruttner F.et H., I955. Von der Paarung der Honigbiene. L. Bien., 3 (I), I-28

Courtois G., Lecomte J., 1958. Sur un procédé de marquage des abeilles butineuses au moyen d'un radio-isotope. C. R. Acad. Sci., 247, 147-149.

Courtois G., Lecomte J., I959. Sur la résistance au rayonnement gamma de l'abeille ouvrière. Ann. Abeille, 4, $285-290$.

Courtois G., Lecomte J., Salleron F., ig6r. Étude des échanges de nourriture à l'intérieur de la ruche entre les abeilles ouvrières Apis mellifica. C. R. Acad. Sci., 262, 1057-1059.

FreE J. B., I957. 'The transmission of food between worker honey bees. British Joum. An. Behaviour., 2.

Frisch Von K., r948. Aus dem Leben der Bienen. Wien, Springer Verlag.

Gosswald K., Kloft W., I958. Radioactive Isotope zur Erforschung des Stastenlebens der Inzekten . Umschau, 24, 743-745.

Kalifman J., 1955. Les Abeilles. - Les conquêtes de l'apidologie - la biologie de la ruche. Ed. de Langues Etrangeres, 248-249.

Lecomte J., г96r. Le comportement agressif des ouvrières d'Apis mellifica L. Ann. Abeille, 4, $\mathrm{n}^{\circ} 3$.

Mathis M., I96r. Technique pour le maniement et la conservation des faux-bourdons en dehors de la ruche. C. R. Acad. Sci., 252, $4198-4200$.

Mindt B., I962. Untersuchungen über des Leben des Drohnen, insbesondere emahrung und Geschlechtsreife. Zeits, für Bienent., 9-33.

Nixon H. L., Ribbands C. R., 1952. Food transmission within the honeybee community. Proc. Roy. Soc. B., 150, 42-50.

Oertel E., Emerson R. B., Wheeler H. E., I953. Transfer of radioactivity from worker to drone honey bees after ingestion of radioactive sucrose. Ann. Entom. Soc. America, 46, n' 4 .

PaIN J., 196r. Thèse. Sur la phérormone des reines d'abeilles et ses effets physiologiques. Ann. Abeille, 4, $\mathrm{n}^{\circ} 2,73^{-1} 5^{2}$.

Roubaud E. , I950. La désocialisation chez l'abeille domestique (Apis mellifica). Coll. Int. C. N.R. S., Paris, 34, $293^{-295}$. 